\title{
On the Power Performance of Test Statistics for the Generalized Rayleigh Interval Grouped Data
}

\author{
Hatim Solayman Migdadi \\ Department of Mathematics, Faculty of Science and Information Technology, Jadara University, Irbid, Jordan \\ Email: hmigdadi@jadara.edu.jo
}

Received 16 July 2015; accepted 23 August 2015; published 26 August 2015

Copyright (C) 2015 by author and Scientific Research Publishing Inc. This work is licensed under the Creative Commons Attribution International License (CC BY). http://creativecommons.org/licenses/by/4.0/ (c) (i) Open Access

\begin{abstract}
In this paper, the weighted Kolmogrov-Smirnov, Cramer von-Miss and the Anderson Darling test statistics are considered as goodness of fit tests for the generalized Rayleigh interval grouped data. An extensive simulation process is conducted to evaluate their controlling of type 1 error and their power functions. Generally, the weighted Kolmogrov-Smirnov test statistics show a relatively better performance than both, the Cramer von-Miss and the Anderson Darling test statistics. For large sample values, the Anderson Darling test statistics cannot control type 1 error but for relatively small sample values it indicates a better performance than the Cramer von-Miss test statistics. Best selection of the test statistics and highlights for future studies are also explored.
\end{abstract}

\section{Keywords}

Generalized Rayleigh Distribution, Interval Grouped Data, Goodness of Fit Tests, Empirical Type 1 Error, Power Function

\section{Introduction}

In many real practical applications, when it is not feasible to have a complete data for statistical inference about the hypothesized statistical model, grouped data arise frequently in many fields of economics, medicine, engineering and variety branches of science. In survival and reliability analysis, performing industrial life testing experiments by continuous monitoring the test units may incorporate an error measurements in some failure units, tediously, costly and time consuming in many situations. Therefore, it is more convenient to inspect the test units intermittently for failure by initially dividing the time scale line into adjacent intervals by constant inspection times $t_{j}, j=1,2, \cdots, k$ to have the interval grouped which mainly consists of the numbers of failure 
units in the given intervals. Having the interval grouped data from the continuous lifetime model may override the testing settings but increases the efforts needed for making any statistical inference. Such type of data is considered by many authors as in Pipper and Ritz [1], Aludaat [2] and Migdadi and Al-Batah [3].

Many researchers have proposed and modified test statistics for fitting grouped data to the hypothesized statistical distributions. Initially, the Chi Square test statistic proposed by Pearson [4] is mainly considered. This statistic is based on the discrepancies between the observed and the expected frequencies in the given intervals. Further modifications of the Chi Square test statistic are studied by many authors, as in Best and Rayner [5] [6].

The initial statistic for goodness of fit test is $\mathrm{CH}$ square, then other statistics are considered as a distance between the theoretical and empirical distribution, for details see ref [10]. Test statistics are derived from the sum of discrepancies between the empirical and the hypothetical distribution functions. Among these statistics are the Kolmogrov- Smirnov, Cramer von-Miss and Anderson Darling test statistics. Choulakian [7] modified these statistics for testing a discrete distribution. Spinelli and Stephens [8] have used these statistics for testing the poisson distribution. Spinelli [9] has considered these statistics for testing grouped data fit to the exponential distribution. Baklizi [10] proposed the weighted Kolmogorov test statistics for the Rayleigh interval grouped data. Many other researchers studied the asymptotic distributions of some of these statistics as in Schmid [11] and Pettitt and Stephens [12]. Modifications, critical values and powers of these statistics are also considered for some distributions with grouped data as in Conover [13], Reidwyl [14], Maag [15], Damianou and Kemp [16], Gulati and Neus [17], Richard and Lockhart [18], and Ampai and Kanisa [19].

As an extension to the Rayleigh distribution, the generalized Rayleigh distribution is used for a more general lifetime data. The probability distribution, the cumulative distribution and the reliability functions of the generalized Rayleigh distribution with scale parameter $\theta$ and shape parameter $\beta$ are given respectively by

$$
\begin{aligned}
& f(t, \theta, \beta)=2 \beta \theta \exp \left(-\theta t^{2}\right)\left(1-\exp \left(-\theta t^{2}\right)\right)^{\beta} \\
& F(t, \theta, \beta)=\left(1-\exp \left(-\theta t^{2}\right)\right)^{\beta} \\
& R(t, \theta, \beta)=1-\left(1-\exp \left(-\theta t^{2}\right)\right)^{\beta}
\end{aligned}
$$

where: $t \geq 0, \theta>0, \beta>0$.

Raqab and Kundu [20] showed that this lifetime model can be widely used in survival and reliability analysis. Maximum likelihood estimators for both the scale parameter $\theta$ and the shape parameter $\beta$ based on the interval grouped data are obtained by Debasis and Raqabb [21].

The aim of this study is to evaluate performance of the weighted Kolmogrov-Smirnov and the modified Cramer von-Miss and Anderson Darling test statistics for fitting the interval grouped data to the generalized Rayleigh distribution. The test statistics are compared in terms of their powers and controlling of type 1 errors. In the next section the test statistics are derived using the interval grouped data. In Section 3 an extended simulation study is conducted with the original generalized Rayleigh distribution data to find the test statistics that control type 1 error. In Section 4 an alternative data from other lifetime distributions are used in connection with the simulation study to obtain powers of the given test statistics. Results from the simulation study are summarized in Section 5 and finally in Section 6 general conclusion and highlights of the overall finding and future works are also involved.

\section{The Test Statistics}

Suppose, we have a random sample of size $n$ from the generalized Rayleigh distribution with probability density function given by (1).

Assume that the time scale line is divided by the inspection points $t_{i}, i=1,2, \cdots, k-1$

Suppose $t_{0}=0, t_{k}=\infty$, then we have the intervals $\left[0, t_{1}\right),\left[t_{1}, t_{2}\right), \cdots,\left[t_{k-1}, \infty\right)$.

Let $f_{i}$ : be the number of failure units in the ith interval, $i=1,2, \cdots, k$, and assume that $\hat{\theta}, \hat{\beta}$ are the maximum likelihood estimators of $\theta, \beta$ based on the above interval grouped data. Then the empirical and the theoretical distribution functions at the inspection times $t_{i}, i=1,2, \cdots, k-1$ are respectively

$$
F_{n}\left(t_{i}\right)=\frac{1}{n} \sum_{j=1}^{i} f_{j}, \quad F\left(t_{i}\right)=\left(1-\exp \left(-\hat{\theta} t^{2}\right)\right)^{\hat{\beta}}
$$


Hence, following Baklizi [10], the weighted Kolomogorov test statistics are given by

$$
\begin{aligned}
& G v 1=\sum_{i=1}^{k-1}\left(F\left(t_{i}\right)\left(1-F\left(t_{i}\right)\right)\right)^{\frac{-1}{2}} S_{i} \\
& G v 2=\sum_{i=1}^{k-1}\left(\frac{k}{2}-i\right)^{2} S_{i} \\
& G v 3=\sum_{i=1}^{k-1} S_{i}
\end{aligned}
$$

where: $S_{i}=\left|F_{n}\left(t_{i}\right)-F\left(t_{i}\right)\right|, i=1,2, \cdots, k-1$.

Setting: the probability of failure in the corresponding intervals: $j=1,2, \cdots, k$

$$
P_{j}(\theta, \beta)=P\left(t_{j-1}<T<t_{j}\right)=R\left(t_{j-1}, \theta, \beta\right)-R\left(t_{j}, \theta, \beta\right)
$$

Then, following Choulakian [7], the modified Anderson Darling test statistics is given by

$$
A d=\frac{1}{n} \sum_{j=1}^{k} \frac{W_{j} P_{j}(\hat{\theta}, \hat{\beta})}{H_{j}\left(1-H_{j}\right)}
$$

where: $H_{j}=\sum_{i=1}^{j} P_{i}(\hat{\theta}, \hat{\beta})$ and $W_{j}=\sum_{i=1}^{j} f_{i}-n \sum_{i=1}^{j} P_{i}(\hat{\theta}, \hat{\beta})$.

And, following Spinelli [9], the modified Cramer test statistics is given by

$$
C v m=\frac{1}{n} \sum_{i=1}^{k} W_{j} P_{j}(\hat{\theta}, \hat{\beta})
$$

\section{Simulation Study}

In this section, an extensive simulation study is conducted to obtain the test statistics that control type 1 error for testing the hypotheses:

$\mathrm{H}_{0}$ : the data distribution is the generalized Rayleigh distribution

$\mathrm{H}_{1}$ : the data distribution is not the generalized Rayleigh distribution

At the significance level: $\alpha=0.05$ with the following indices:

The sample size: $n=30,50,100$

The number of intervals: $k=5,7,10$

The original generalized Rayleigh distribution data with parameters: $\theta=0.05, \beta=0.8$

The inspection times $t_{i}, i=1,2, \cdots, k-1$ are taken to be equally likely spaced.

For each combination, the following steps describe the simulation process:

(1) Generate a random sample of size $n$ from the generalized distribution and group it into $k$ intervals

(2) Compute the values of the MLE's: $\hat{\theta}, \hat{\beta}$ based on the interval grouped data

(3) Compute the values of the test statistics: Gv1, Gv2, Gv3, Ad, Cvm

(4) Generate a bootstrap sample of size $n$ from the generalized Rayleigh distribution with parameters $\hat{\theta}, \hat{\beta}$

and repeat the steps 2 and 3 to have the new values of the test statistics $G v 1^{*}, G v 2^{*}, G v 3^{*}, A d^{*}, C v m^{*}$

(5) Repeat the step 4, $m=500$ times and compute the number of values $h^{*}$ for which the values of the test statistics found in 4 are greater than the test statistics found in 2 and compute the $p$ value for each statistics as: $p=\frac{h^{*}+1}{m+1}$.

(6) Repeat the steps 1-5, 1000 times and compute the empirical type 1 error for each statistics as $\frac{w}{1000}$, where: $w=$ the number of the $p$ values less than the given significance level: $\alpha=0.05$.

Based on the Bradley [22] test, the test statistics is considered to control type 1 error if the corresponding value of its empirical type 1 error is between 0.025 and 0.075 for the significance level $=0.05$. 


\section{Power of the Test Statistics}

To find the empirical power for each of the given test statistics, an alternative non-generalized Rayleigh data are generated in step 1 of the simulation process described in the previous section. Hence, we consider the following distributions:

-One parameter Rayleigh distribution with distribution function:

$$
F(t, \theta)=1-\exp \left(-\theta t^{2}\right), t \geq 0, \theta>0
$$

-Weibull distribution with distribution function:

$$
F(t, \theta, \beta)=1-\exp \left(-\theta t^{\beta}\right), t \geq 0, \theta>0, \beta>0
$$

-Generalized Exponential distribution with distribution function:

$$
F(t, \theta, \beta)=(1-\exp (-\theta t))^{\beta}, t \geq 0, \theta>0, \beta>0
$$

\section{Results and Conclusions}

In this section, found out results about the empirical type 1 error and the power functions of the test statistics are illustrated. Compressions of the test statistics and the affecting factors are also illustrated.

\subsection{Controlling of Type 1 Error}

The empirical type 1 error rates at the significance level $\alpha=0.05$ of the test statistics as applied to the original data are presented in Table 1. It appears clearly that

(1) The test statistics $G v 1, G v 3$ can control type 1 error for any sample size and any number of inspection in-

\begin{tabular}{|c|c|c|c|c|}
\hline \multirow{2}{*}{ Sample size } & \multirow{2}{*}{ Test statistics } & \multicolumn{3}{|c|}{ number of inspection intervals } \\
\hline & & 10 & 7 & 5 \\
\hline \multirow{5}{*}{30} & Gv1 & 0.053 & 0.057 & 0.073 \\
\hline & Gv2 & 0.058 & 0.059 & 0.066 \\
\hline & Gv3 & 0.066 & 0.067 & 0.073 \\
\hline & Cvm & 0.053 & 0.064 & 0.068 \\
\hline & Ad & 0.067 & 0.069 & 0.071 \\
\hline \multirow{5}{*}{50} & Gv1 & 0.049 & 0.065 & 0.072 \\
\hline & Gv2 & 0.050 & 0.057 & 0.061 \\
\hline & Gv3 & 0.065 & 0.063 & 0.071 \\
\hline & Cvm & 0.057 & 0.061 & 0.067 \\
\hline & Ad & 0.061 & 0.068 & 0.062 \\
\hline \multirow{5}{*}{100} & Gv1 & 0.064 & 0.073 & $0.094^{*}$ \\
\hline & Gv2 & 0.047 & 0.058 & 0.063 \\
\hline & Gv3 & 0.048 & 0.057 & 0.058 \\
\hline & Cvm & $0.079^{*}$ & $0.077^{*}$ & $0.076^{*}$ \\
\hline & Ad & 0.057 & 0.059 & 0.061 \\
\hline
\end{tabular}
tervals.

Table 1. Empirical type 1 error rates.

\footnotetext{
${ }^{*}$ Not control type 1 error.
} 
(2) The test statistic $G v 1$ cannot control type 1 error for the sample size $n=100$ and the number of inspection Intervals $k=5$.

(3) The weighted Kolmogrov-Smirnov statistic $G v 2$ dominate $G v 1$ and $G v 3$ when the sample sizes $n=30,50$ and the statistic $G v 3$ is relatively better than $G v 2$ when the sample size $n=100$.

(4) The Anderson Darling test statistic: Ad cannot control type 1 error for the sample size $n=100$ using any number of inspection intervals. But for the sample sizes: $n=30, n=50$ it gives a better controlling of type 1 error than the Cramer von-Miss Cvm test statistic.

(5) Generally, the statistics $G v 1, G v 2$ and $G v 3$ have more controlling of type 1 errors than both Cramer vonMiss and Anderson Darling test statistics.

\subsection{Power Performance}

The powers of the test statistics applied to the nongeneralized Rayleigh grouped data are presented in the tables: Tables 2-7 where we have the following results:

(1) The power functions of the given test statistics increases as the sample size and the number of inspection intervals increases.

(2) For the sample sizes: $n=30$ and $n=50$, the Anderson Darling test statistic have more power than the Cramer von-Miss test statistic.

(3) Among the weighted Kolmogrov-Smirnov statistics, $G v 2$ has the greatest power, next came $G v 3$, and then Gv1.

(4) Generally, the weighted Kolmogrov-Smirnov test statistics have greater power than the Anderson Darling and the Cramer von-Miss test statistics. Except at the sample size 30, the Anderson Darling test statistics gives greater power than $G v 1$ when the alternative data are considered from the from: the one parameter Rayleigh distribution with scale parameter $\theta=0.85$, the Weibull distribution with scale Parameter $\theta=0.65$ and shape parameter $\rho=1.8$ and the generalized exponential distribution with scale parameter $\theta=1.5$ and shape parameter $\delta=1$.

Table 2. Power of the test statistics for the interval grouped data from the one parameter Rayleigh distribution with scale parameter $\theta=0.85$.

\begin{tabular}{|c|c|c|c|c|}
\hline \multirow{2}{*}{ Sample size } & \multirow{2}{*}{ Test statistics } & \multicolumn{3}{|c|}{ Number of inspection intervals } \\
\hline & & 5 & 7 & 10 \\
\hline \multirow{5}{*}{30} & $G v 1$ & 0.431 & 0.433 & 0.452 \\
\hline & $G v 2$ & 0.445 & 0.453 & 0.472 \\
\hline & $G v 3$ & 0.439 & 0.442 & 0.461 \\
\hline & $\mathrm{Cvm}$ & 0.440 & 0.451 & 0.468 \\
\hline & Ad & 0.398 & 0.406 & 0.417 \\
\hline \multirow{5}{*}{50} & $G v 1$ & 0.508 & 0.562 & 0.574 \\
\hline & $G v 2$ & 0.512 & 0.567 & 0.583 \\
\hline & $G \vee 3$ & 0.509 & 0.564 & 0.580 \\
\hline & $\mathrm{Cvm}$ & 0.507 & 0.536 & 0.559 \\
\hline & Ad & 0.478 & 0.508 & 0.532 \\
\hline \multirow{5}{*}{100} & $G v 1$ & 0.672 & 0.693 & 0.703 \\
\hline & $G v 2$ & 0.684 & 0.721 & 0.755 \\
\hline & $G \vee 3$ & 0.677 & 0.715 & 0.734 \\
\hline & $\mathrm{Cvm}$ & 0.662 & 0.691 & 0.701 \\
\hline & $A d$ & 0.673 & 0.702 & 0.718 \\
\hline
\end{tabular}


Table 3. Power of the test statistics for the interval grouped data from the one parameter Rayleigh distribution with scale parameter $\theta=0.05$.

\begin{tabular}{|c|c|c|c|c|}
\hline \multirow{2}{*}{ Sample size } & \multirow{2}{*}{ Test statistics } & \multicolumn{3}{|c|}{ Number of inspection intervals } \\
\hline & & 5 & 7 & 10 \\
\hline \multirow{5}{*}{30} & $G v 1$ & 0.602 & 0.612 & 0.703 \\
\hline & $G v 2$ & 0.635 & 0.658 & 0.741 \\
\hline & $G v 3$ & 0.619 & 0.635 & 0.727 \\
\hline & $\mathrm{Cvm}$ & 0.576 & 0.598 & 0.633 \\
\hline & Ad & 0.518 & 0.542 & 0.579 \\
\hline \multirow{5}{*}{50} & $G v 1$ & 0.687 & 0.771 & 0.803 \\
\hline & $G v 2$ & 0.712 & 0.796 & 0.825 \\
\hline & Gv3 & 0.705 & 0.782 & 0.811 \\
\hline & Cvm & 0.642 & 0.679 & 0.682 \\
\hline & Ad & 0.639 & 0.665 & 0.674 \\
\hline \multirow{5}{*}{100} & $G v 1$ & 0.732 & 0.799 & 0.853 \\
\hline & $G v 2$ & 0.803 & 0.867 & 0.902 \\
\hline & Gv3 & 0.794 & 0.855 & 0.902 \\
\hline & $\mathrm{Cvm}$ & 0.652 & 0.688 & 0.704 \\
\hline & $A d$ & 0.727 & 0.796 & 0.815 \\
\hline
\end{tabular}

Table 4. Power of the test statistics for the interval grouped data from the Weibull distribution with scale parameter $\theta=0.65$, and shape parameter $\beta=1.8$.

\begin{tabular}{|c|c|c|c|c|}
\hline \multirow{2}{*}{ Sample size } & \multirow{2}{*}{ Test statistics } & \multicolumn{3}{|c|}{ Number of inspection intervals } \\
\hline & & 5 & 7 & 10 \\
\hline \multirow{5}{*}{30} & $G v 1$ & 0.483 & 0.501 & 0.513 \\
\hline & $G v 2$ & 0.522 & 0.546 & 0.581 \\
\hline & Gv3 & 0.517 & 0.529 & 0.564 \\
\hline & $\mathrm{Cvm}$ & 0.519 & 0.536 & 0.579 \\
\hline & Ad & 0.476 & 0.500 & 0.507 \\
\hline \multirow{5}{*}{50} & $G v 1$ & 0.632 & 0.672 & 0.740 \\
\hline & $G v 2$ & 0.671 & 0.703 & 0.776 \\
\hline & Gv3 & 0.656 & 0.698 & 0.754 \\
\hline & $\mathrm{Cvm}$ & 0.603 & 0.651 & 0.698 \\
\hline & Ad & 0.584 & 0.622 & 0.679 \\
\hline \multirow{5}{*}{100} & $G v 1$ & 0.723 & 0.758 & 0.804 \\
\hline & $G v 2$ & 0.761 & 0.790 & 0.813 \\
\hline & Gv3 & 0.756 & 0.778 & 0.808 \\
\hline & Cvm & 0.632 & 0.677 & 0.715 \\
\hline & Ad & 0.722 & 0.746 & 0.796 \\
\hline
\end{tabular}


Table 5. Power of the test statistics for the interval grouped data from the Weibull distribution with scale parameter $\theta=0.05$, and shape parameter $\beta=0.3$.

\begin{tabular}{|c|c|c|c|c|}
\hline \multirow{2}{*}{ Sample size } & \multirow{2}{*}{ Test statistics } & \multicolumn{3}{|c|}{ Number of inspection intervals } \\
\hline & & 5 & 7 & 10 \\
\hline \multirow{5}{*}{30} & $G v 1$ & 0.652 & 0.671 & 0.712 \\
\hline & $G v 2$ & 0.692 & 0.703 & 0.755 \\
\hline & Gv3 & 0.674 & 0.692 & 0.738 \\
\hline & Cvm & 0.579 & 0.633 & 0.661 \\
\hline & $A d$ & 0.568 & 0.621 & 0.646 \\
\hline \multirow{5}{*}{50} & $G v 1$ & 0.733 & 0.802 & 0.864 \\
\hline & $G v 2$ & 0.762 & 0.836 & 0.907 \\
\hline & $G v 3$ & 0.744 & 0.821 & 0.895 \\
\hline & Cvm & 0.712 & 0.792 & 0.852 \\
\hline & Ad & 0.710 & 0.775 & 0.791 \\
\hline \multirow{5}{*}{100} & $G v 1$ & 0.862 & 0.883 & 0.911 \\
\hline & $G v 2$ & 0.865 & 0.902 & 0.932 \\
\hline & Gv3 & 0.864 & 0.897 & 0.926 \\
\hline & Cvm & 0.724 & 0.798 & 0.853 \\
\hline & $A d$ & 0.767 & 0.832 & 0.861 \\
\hline
\end{tabular}

Table 6. Power of the test statistics for the interval grouped data from the generalized exponential distribution with scale parameter $\theta=1.5$ and shape parameter $\beta=1$.

\begin{tabular}{|c|c|c|c|c|}
\hline \multirow{2}{*}{ Sample size } & \multirow{2}{*}{ Test statistics } & \multicolumn{3}{|c|}{ Number of inspection intervals } \\
\hline & & 5 & 7 & 10 \\
\hline \multirow{5}{*}{30} & $G v 1$ & 0.512 & 0.518 & 0.523 \\
\hline & Gv2 & 0.534 & 0.561 & 0.589 \\
\hline & $G v 3$ & 0.528 & 0.543 & 0.572 \\
\hline & Cvm & 0.531 & 0.552 & 0.581 \\
\hline & Ad & 0.507 & 0.511 & 0.518 \\
\hline \multirow{5}{*}{50} & Gv1 & 0.653 & 0.679 & 0.748 \\
\hline & $G v 2$ & 0.692 & 0.721 & 0.792 \\
\hline & $G v 3$ & 0.684 & 0.709 & 0.765 \\
\hline & Cvm & 0.620 & 0.652 & 0.702 \\
\hline & Ad & 0.611 & 0.637 & 0.688 \\
\hline \multirow{5}{*}{100} & $G v 1$ & 0.752 & 0.771 & 0.810 \\
\hline & $G v 2$ & 0.773 & 0.803 & 0.851 \\
\hline & Gv3 & 0.772 & 0.796 & 0.835 \\
\hline & Cvm & 0.631 & 0.662 & 0.710 \\
\hline & Ad & 0.658 & 0.694 & 0.757 \\
\hline
\end{tabular}


Table 7. Power of the test statistics for the interval grouped data from the generalized exponential distribution with scale parameter $\theta=0.05$ and shape parameter $\beta=2.5$.

\begin{tabular}{|c|c|c|c|c|}
\hline \multirow{2}{*}{ Sample size } & \multirow{2}{*}{ Test statistics } & \multicolumn{3}{|c|}{ Number of inspection intervals } \\
\hline & & 5 & 7 & 10 \\
\hline \multirow{5}{*}{30} & $G v 1$ & 0.681 & 0.693 & 0.732 \\
\hline & $G v 2$ & 0.703 & 0.721 & 0.758 \\
\hline & Gv3 & 0.695 & 0.713 & 0.755 \\
\hline & Cvm & 0.618 & 0.657 & 0.703 \\
\hline & Ad & 0.615 & 0.632 & 0.688 \\
\hline \multirow{5}{*}{50} & $G v 1$ & 0.784 & 0.815 & 0.866 \\
\hline & $G v 2$ & 0.801 & 0.838 & 0.913 \\
\hline & Gv3 & 0.792 & 0.831 & 0.907 \\
\hline & Cvm & 0.727 & 0.793 & 0.857 \\
\hline & Ad & 0.714 & 0.781 & 0.796 \\
\hline \multirow{5}{*}{100} & $G v 1$ & 0.868 & 0.887 & 0.923 \\
\hline & $G v 2$ & 0.875 & 0.903 & 0.935 \\
\hline & $G v 3$ & 0.872 & 0.901 & 0.928 \\
\hline & Cvm & 0.728 & 0.794 & 0.861 \\
\hline & $A d$ & 0.751 & 0.835 & 0.867 \\
\hline
\end{tabular}

(5) There is a significant affection in the power of the test statistics in fitting the generalized Rayleigh distribution with shape parameter $\delta \neq 1$ for the lifetimes data. This affection clearly appears when using the alternatives: the one parameter Rayleigh and the generalized exponential distributions

(6) The powers of the test statistics are mainly affected by the parameters of the alternative distributions, when the alternative Weibull distribution with scale parameter $\theta=0.65$ and shape parameter $\rho=1.8$ is considered, the values of the power functions are strictly less than their corresponding values when the alternative is the Weibull distribution with scale parameter $\theta=0.05$ and shape parameter $\rho=3$.

A possible explanation for this is the degree of similarity between the Weibull distribution and the Generalized Rayleigh distribution when using a complete data at the indicated parameters.

\section{Conclusion and Highlights for Future Work}

This study explored the performance of goodness of fit test statistics for the generalized Rayleigh distribution. Generally, the weighted Kolmogrov-Smirnov test statistics have a relatively better performance in controlling type 1 error and in the power functions than the modified Cramer von-Miss and Anderson Darling test statistics. As it cannot control type 1 error when the sample size $n=100$, the Anderson Darling test has more power than the Cramer von-Miss and the weighted Kolmogrov-Smirnov Gv1 test statistics when the sample size $n=30$ or $n$ $=50$. This indicates that the researcher has to take into account both the sample size and number of inspection intervals when choosing the test statistic for fitting the interval grouped data to the generalized Rayleigh distribution. Future works may involve other lifetime models in the presence of censoring schemes within the intervals. Critical regions for the test statistics at different significance levels can also be a subject of concern.

\section{References}

[1] Pipper, C.B. and Ritz, C. (2006) Cheking the Grouped Data Version of Cox Model for Interval Grouped Survival Data. Scandinavian Journal of Statistics, 10, 1467-1469. 
[2] Aludaat, K.M., Alodat, M.T. and Alodat, T.T. (2008) Parameter Estimation of Burr Type X Distribution for Grouped Data. Applied Mathematical Sciences, 2, 415-423.

[3] Migdadi, H.S. and Al-Batah, M.S. (2014) Bayesian Inference Based on the Interval Grouped Data from the Weibull Model with Application. British Journal of Mathematics \& Computer Science, 4, 1170-1183. http://dx.doi.org/10.9734/BJMCS/2014/7930

[4] Pearson, K. (1900) On a Criterion That a Given System of Deviations from the Probable in the Case of a Correlated System of Variables Is Such That It Can Be Reasonably Supposed to Have Arisen from Random Sampling. Philosophical Magazine, 50, 157-175. http://dx.doi.org/10.1080/14786440009463897

[5] Best, D.J. and Rayner, J.C.W. (2007) Chi Squared Components for Tests of Fit and Improved Models for the Grouped Exponential Distribution. Computational Statistics and Data Analysis, 51, 3946-3954. http://dx.doi.org/10.1016/j.csda.2006.03.014

[6] Best, D.J. and Rayner, J.C.W. (2006) Improved Testing for the Binomial Distribution Using Chi Squared Components with Data Dependent Cells. Journal of Statistical Computation and Simulation, 76, 75-81. http://dx.doi.org/10.1080/00949650412331320891

[7] Choulakian, V., Lockhart, R.A. and Stephens, M.A. (1994) Cramer-von Mises Test of Discrete Distribution. The Canadian Journal of Statistics, 22, 125-137. http://dx.doi.org/10.2307/3315828

[8] Spinelli, J.J. and Stephens, M.A. (1997) Cramer-von Mises Tests of Fit for the Poisson Distribution. The Canadian Journal of Statistics, 25, 257-268. http://dx.doi.org/10.2307/3315735

[9] Spinelli, J.J. (2001) Testing of Fit for the Grouped Exponential Distribution. The Canadian Journal of Statistics, 29, 451-458. http://dx.doi.org/10.2307/3316040

[10] Baklizi, A. (2006) Weighted Kolmogrov-Simirnov Type Tests for Grouped Rayleigh Data. Applied Mathematical Modeling, 30, 437-445. http://dx.doi.org/10.1016/j.apm.2005.05.012

[11] Schmid, P. (1988) On the Kolmogrov and Smirnov Limit Theorems for Discontinuous Distribution Functions. The Annals of Mathematical Statistics, 29, 1011-1027. http://dx.doi.org/10.1214/aoms/1177706438

[12] Pettitt. A.N. and Stephens. M.A. (1977) The Kolmogrov-Smirnov Goodness-of-Fit Statistic with Discrete and Grouped Data. Technometrics, 19, 205-210. http://dx.doi.org/10.1080/00401706.1977.10489529

[13] Conover, W.J. (1992) A Kolmogrov Goodness-of-Fit Test for Discontinuous Distributions. Journal of the American Statistical Association, 6, 591-596. http://dx.doi.org/10.1080/01621459.1972.10481254

[14] Reidwyl, H. (1967) Goodness of Fit. Journal of the American Statistical Association, 6, 390-398.

[15] Maag, U.R., Streit. P. and Drouilly, P.A. (1973) Goodness-of-Fit Test for Grouped Data. Journal of the American Statistical Association, 68, 462-465. http://dx.doi.org/10.1080/01621459.1973.10482456

[16] Damianou, C. and Kemp, A.W. (1990) New Goodness of Fit Statistics for Discrete and Continuous Data. American Journal of Mathematics, and Management Science, 10, 275-307.

[17] Gulati, S. and Neus, J. (2003) Goodness of Fit Statistics for the Exponential Distribution When the Data Are Grouped. Communications in Statistics, Theory and Methods, 32, 681-700. http://dx.doi.org/10.1081/STA-120018558

[18] Richard, A., Lockhart, J., Spinelli, J.J. and Stephens, M.A. (2007) Cramér-Von Mises Statistics for Discrete Distributions with Known Parameters. The Canadian Journal of Statistics, 35, 125-133.

[19] Ampai, T. and Kanisa, C. (2011) A Power of Comparison of Goodness of Fit Tests for Exponential Distribution with Grouped Data. Thailand Statistician, 9, 37-49.

[20] Raqab, M.Z. and Kundu, D. (2006) Burr Type X Distribution: Revisited. Journal of Probability and Statistical Sciences, 4, 179-193.

[21] Kundua, D. and Raqabb, M.Z. (2005) Generalized Rayleigh Distribution. Different Methods of Estimations. Computational Statistics \& Data Analysis, 49, 187-200.

[22] Bradley, J.V. (1978) Robustness. British Journal of Mathematics and Statistical Psychology, 31, $144-151$. http://dx.doi.org/10.1111/j.2044-8317.1978.tb00581.x 